\title{
Advances in the study of the biological function of Daxx
}

\author{
Jinhong Liu ${ }^{1 *}$, Guozuo Xiong ${ }^{2}$, Duanfang Liao ${ }^{1,3}$, and Qinhui Tuo ${ }^{1}$ \\ ${ }^{1}$ Learning Key Laboratory for Pharmacoproteomics, School of Life Science and Technology, University of South China, \\ Hengyang 421001, China. \\ ${ }^{2}$ Department of General Surgery, The Second Affiliated Hospital in University of South China, Hengyang 421001, China. \\ ${ }^{3}$ Department of Traditional Chinese Diagnotics, School of Pharmacy, Hunan University of Chinese Medicine, Changsha, \\ 410208. \\ Accepted 10 March, 2012

\begin{abstract}
Death domain-associated protein (Daxx) was originally identified as a protein that specifically binds to the death domain of the transmembrane death receptor Fas (also called CD95) in the cytoplasm and potentiates Fas-induced apoptosis. Over expression of Daxx enhances Fas-mediated apoptosis and activates the Jun N-terminal kinase (JNK) pathway. Daxx was found in the nuclears where it localizes to promyelocytic leukaemia (PML) oncogenic domains (PODs). As a highly conserved nuclear protein, Daxx plays an important role in proapoptosis, antiapoptosis and transcriptional regulation. This article reviews the latest advances in the study of the biological function of Daxx.
\end{abstract}

Key words: Death domain-associated protein (Daxx), cell apoptosis, transcriptional regulation, biological function.

\section{INTRODUCTION}

As a highly conserved nuclear protein, Death domainassociated protein (Daxx) was originally identified as a protein that specifically binds to the death domain of the transmembrane, death receptor FAS (also called CD95) in the cytoplasm and potentiates FAS-induced apoptosis (Yang et al., 1997). A large proportion of Daxx molecules are nuclear, however, associate with the promyelocytic leukaemia (PML) nuclear body (PML-NB) and other subnuclear domains. Daxx has been suggested to function as a pro-apoptotic protein downstream of FAS through activation of the c-Jun-N-terminal kinase (JNK) pathway in a FADD-independent manner (Yang et al., 1997). As a transcriptional regulator, Daxx regulates the transcription of a variety of factors and expresses different biological effects.

In recent years, both domestic and foreign researchers took a lot of research and explorations of Daxx, which were about apoptosis, anti-apoptosis and transcriptional regulation. This paper reviews the latest advances in the study of the biological function of Daxx.

\section{THE BIOLOGICAL CHARACTERISTICS OF DAXX}

Daxx is a highly conserved protein. Human Daxx protein is composed by 740 amino acids and its molecular weight is $81.4 \mathrm{KD}$. Mouse Daxx protein is composed of 739 amino acids, and its molecular weight is $81.3 \mathrm{kD}$. Daxx between the human and murine proteins has $69 \%$ identity. The total length of Daxx gene is about $315 \mathrm{~kb}$ and it has 7 exons and 6 introns. Because of the different post-transcriptional modification, Daxx molecules have three forms of existence, their molecular weight were 70 , 97 and $120 \mathrm{ku}$, respectively. Daxx contains four regions, 
which are two N-terminal paired amphipathic helices (PAHs); a coiled-coil domain; an acidic region and a Cterminal serine/proline/threonine rich region. These structure domains make close association with transcription of Daxx.

Daxx is ubiquitously expressed throughout the body, such as liver, kidney, heart, 293 cells, HeLa cell, etc. In addition, Daxx is also expressed in chronic lymphocytic leukemia (CLL) (Alkan et al., 2005).

\section{DAXX AND PROAPOPTOSIS}

Daxx was initially identified as a protein associated with the death domain of the Fas receptor and involved in Fas-mediated apoptosis as well as the activation of the JNK pathway. However, Daxx-mediated apoptosis signaling pathway, at present, is still not entirely clear. Confirming the initial yeast two-hybrid screen, glutathione S-transferase (GST) pull-down experiments showed the C-terminal portion of Daxx as interacting with the Fas receptor (YANG et al., 1997).

In order to further investigate the mechanism of Daxx, yeast two-hybrid technique was used to screen the intracellular proteins interacting with Daxx (Liu et al., 2012), and 13 positive colonies and three proteins interacting with Daxx were obtained. One of the candidate proteins was identified as ferritin, heavy polypeptide 1 (FTH1). The interaction between Daxx and FTH1 was further supported by GST pull-down and co-immunoprecipitation, respectively. Then, Daxx was determined to induce apoptosis through the Fas-Daxx-ASK1-JNK1 signaling pathway. Schepers et al. (2005) found out that an inhibitory role of HSP27 in VP-16-mediated activation of the ASK1/p38/JNK pathway after HSP27 combine with Daxx, which is in the monocytic acute myeloblastic leukemia (AML). As a highly conserved nuclear protein, Daxx deletion mutant (aa 501 to 625) has been known to be an inducer of apoptosis, and Song et al. (2004) observed that the Bax-dependent mitochondrial death signaling pathway plays an important role in Daxx 501 to 625-induced apoptosis. What is more, Daxx 501 to 625induced apoptosis is mediated through the ASK1-MEKJNK/p38-Bim-Bax-dependent caspase pathway.

Muromoto et al. (2010) found out that knock down of Daxx attenuated lactate dehydrogenase leakage from cells, indicating that Daxx positively regulates cell death during gp130/STAT3-mediated cell proliferation.

In exploring whether Daxx mediates oxidized lowdensity lipoprotein (Ox-LDL)-induced apoptosis in macrophage, He et al. (2010) found out that Ox-LDL upregulated the expression of Daxx mRNA and caveolin-1, increased the accumulation of intercellular cholesterol and induced the apoptosis of RAW264.7 macrophages. However, Ox-LDL-induced intercellular cholesterol accumulation and apoptosis in RAW264.7 cells were prevented by Daxx siRNA. Ox-LDL also induced caveolin-1 expression and this effect was significantly suppressed by Daxx siRNA. It can be concluded that Daxx mediates Ox-LDL-induced cholesterol accumulation and apoptosis in macrophages by up-regulating caveolin1 expression. We found out that over-expression of Daxx facilitated HepG2 cells apoptosis induced by hydrogen peroxide (Tuo et al., 2008). Furthermore, there may be a synergetic relation with apoptosis and increase of JNK activity. In order to elucidate the functional relevance of Daxx in apoptosis signaling of malignant lymphocytes, Jurkat T-cells were stably transfected with a Daxxexpressing vector or with the respective Daxx-negative control vector. Boehrer et al. (2005) demonstrated that ectopic expression of Daxx substantially increased the rate of apoptosis upon incubation with death receptor agonists such as Fas and TRAIL, as well as upon incubation with the cytotoxic drug doxorubicin (DOX). Although, enforced apoptosis caused by ectopic Daxx expression is caspase-dependent in both cases, major differences between Fas/Tumor necrosis factor-related apoptosis-inducing ligand (TRAIL)-induced apoptosis and doxorubicin-induced apoptosis are observed in the expression patterns of X-linked inhibitor of apoptosis (XIA-P), p53, Bid, ZIP kinase and prostate apoptosis response gene 4 (Par-4). They outlined the major molecular mechanisms underlying the apoptosispromoting effect of Daxx in neoplastic lymphocytes and demonstrated fundamental molecular differences elicited by the overexpression of Daxx, in the extrinsic and intrinsic signaling pathways. Sodium hydrogen exchange isoform 1 (NHE1) (Jung et al., 2008) was identified as a Daxx-interacting protein. During ischemic stress, Daxx translocates from the nucleus to the cytoplasm, where it colocalizes with NHE1. Daxx binds to the ezrin/radixin/moesin-interacting domain of NHE1, in competition with ezrin.

As most studies on the role of Daxx in cell death have been conducted using tumour cell lines, Khelifi et al. (2005) analyzed the function of Daxx in physiological settings. They employed RNA interference to down regulate Daxx in primary fibroblasts. Remarkably, Daxxdepleted cells are resistant to cell death induced by both ultraviolet (UV) irradiation and oxidative stress. Furthermore, the down regulation of Daxx results in impaired MKK/JNK activation. This is the evidence that Daxx promotes cell death and JNK activation in physiological conditions.

\section{DAXX AND ANTIAPOPTOSIS}

Despite the reports advocating a pro-cell-death function for Daxx, several studies have suggested a potential antiapoptotic function for Daxx.

Michaelson and Leder. (2003) found out that Daxx was essential to the embryonic development and to be antiapoptotic, based on the result those large quantities of embryonic stem cells apoptosis when Daxx gene was knocked out from the mouse embryo. The HeLa cell 
sensitize to apoptosis in Daxx-knockout HeLa cell. Daxx can exert the anti-apoptotic function through regulating the two targets in Daxx, NF KB and E2F1.

Chen and Chen (2003) investigated the role of Daxx in Fas- and stress-induced apoptosis by small interfering RNA-mediated Daxx silencing in mammalian cells, and found out that Daxx silences strongly sensitized cells to Fas- and stress-induced apoptosis. These data strongly suggest that Daxx may inhibit Fas and stress-mediated apoptosis by suppressing pro-apoptotic gene expression outside of PML domains. Chaudhary et al. (2010) demonstrated that 4-hydroxynonenal (HNE) modification induced phosphorylation of Daxx at Ser668 and Ser671 to facilitate its cytoplasmic export, which consistently, induced apoptosis in HepG2 cells. However, the use of small interfering RNA-mediated Daxx silencing could induce apoptosis in HepG2 cells, these aspects indicated a potential antiapoptotic function for Daxx. Cermák et al. (2002) showed that over expression of Daxx inhibited anti-CD43 monoclonal antibody (mAb) MEM-59-induce apoptosis of the myeloid progenitor-derived cell line TF-1. Zhang et al. (2010) discovered that down-regulation of MDM2 in acute lymphocytic leukemia cells by berberine occurred at a posttranslational level through modulation of Daxx, which disrupted the MDM2-DAXX-HAUSP interactions and thereby promoted MDM2 selfubiquitination and degradation. These results suggest a potential anti-apoptotic function for Daxx.

In human cells, Daxx interacted with heat shock factor 1 (HSF1) essentially only during stress, that is, when factor trimerization occurred. Daxx is an important mediator of HSF1 activation. Boellmann et al. (2004) suggest a model in which Daxx released from its nuclear stores during stress, opposes repression of HSF1 transactivation competence by multichaperone complex through its interaction with trimerized HSF1. Over expressed HSF1, known to be largely trimeric, only marginally increased HSF1 activity on its own, but potentiated the activating effect of Daxx overexpression.

Zobalova et al. (2008) found out that exposure of cultured cardiomyocyte-like cells to oxidative stress or simulated hypoxia increased the level of accumulated reactive oxygen species (ROS) and apoptosis. Under the conditions of sub-apoptotic stimulation of cardiac myocytes, there was no increase in the level of the Daxx protein, but its translocation from the nucleus to the cytoplasm was increased. Daxx over expression protect the cells from apoptosis, while they are sensitized to cell death following its down-regulation by siRNA. Moreover, lowering the level of the Daxx protein sensitized cardiac myocytes to spontaneous apoptosis, suggesting that the protein may also have a pro-survival role under physiological conditions. Daxx plays a protective role in cultured cardiomyocyte-like cells, at least under the conditions used.

The small ubiquitin-related modifiel-1 (SUMO)-1 is an important posttranslational regulator of different signaling pathways and is involved in the formation of promyelocytic leukemia (PML) protein nuclear bodies (NBs). Meinecke et al. (2007) showed that the increased expression of SUMO-1 in rheumatoid arthritis (RA) synovial fibroblasts (SFs) contributes to the resistance of these cells against Fas-induced apoptosis through increased sumoylation of nuclear PML protein and increased recruitment of the transcriptional repressor Daxx to PML NBs.

\section{DAXX AS A TRANSCRIPTIONAL REGULATOR}

Daxx has been shown to function as a transcriptional regulator via phosphorylation or its interaction with various cytoplasmic and nuclear proteins. Daxx is unlocated in PODs in the absence of PML or under normal circumstances, a condition in which PODs are destroyed. Daxx can localize to regions of condensed chromatin, when Daxx interacts with several transcription factors, including Pax3 (Lehembre et al., 2001), ETS1 (La et al., 2004). Daxx can inhibit their transcription activity, thus gene transcription is repressed and the cell apoptosis does not occur. In B cell lines, Daxx binding to transcription factor Pax 5 and activating transcription of $B$ cells shows the role of transcriptional co-activator. Park et al. (2007) found out that physical interaction between Daxx and p65 provides a functional framework for the inhibition of p65 acetylation by p300/CBP and subsequent repression of NF-KB transcriptional activity.

Daxx functions as a negative AR co-regulator through direct protein-protein interactions. Studies by Lin et al. (2004) revealed that the binding between Daxx and the DNA-binding domain of the ubiquitination AR shows marked suppression on the activity of the AR DNAbinding both in vitro and in vivo. Moreover, ubiquitination of $A R$ at its amino-terminal domain is involved in Daxx interaction and trans-repression.

Chang et al. (2005) observed that Daxx suppresses Smad4-mediated transcriptional activity by direct interaction with the ubiquitination Smad4 and identified a novel role of Daxx in regulating TGF- $\beta$ signaling pathway. Daxx also suppressed STAT3-mediated transcriptional activation, while over expression of Daxx inhibited IL6/STAT3-mediated gene expression (Kenji et al., 2007). Boellmann et al. (2004) found that over expression of Daxx enhanced basal transcription activity of HSF1 in the condition of a stress. Zhang et al (2010) found out that the transcriptional inhibition of HSF4b is associated with Daxx. HSF4b can interact with Daxx in the nucleus and their association is regulated by the phosphorylation of HSF4b/S299. Thus, we can know the binding between Daxx and different transcription factor or related protein can influence the transcription.

Several recent studies have indicated that Daxx is a mediator of lymphocyte death and/or growth suppression, although, the detailed mechanism is unclear. Muromoto 
et al. (2010) found out that Daxx suppresses the gp130/STAT3-dependent cell growth and that Daxx endogenously interacts with STAT3 and inhibits the DNAbinding activity of STAT3. Moreover, small-interfering RNA-mediated knockdown of Daxx enhanced the expression of STAT3-target genes and accelerated the STAT3-mediated cell cycle progression. In addition, knockdown of Daxx-attenuated lactate dehydrogenase leakage from cells, indicating that Daxx positively regulates cell death during gp130/STAT3-mediated cell proliferation.

\section{CONCLUSION AND PROSPECTS}

Currently, the precise function of Daxx in cell death and transcriptional regulation is not fully understood. While apoptosis appears to be an underlying theme in many of the Daxx studies, the role of Daxx in preventing and/or inducing apoptosis is as yet unclear. However, several studies had indicated that Daxx played significant roles in proapoptosis, antiapoptosis and transcriptional regulation. Certainly, the biochemical function and mechanism of Daxx are in progress and will be critical in further elucidating the functional role of Daxx. In addition, it will bring some novel ideas in prevent and tie at viral infection, cancer, leukemia and cardiovascular disease.

\section{ACKNOWLEDGEMENTS}

This work was supported by The National Natural Science Foundation of China (No.30971267, 30971170 and 30600249) and The Construct program of the key discipline in Hunan Province.

\section{REFERENCES}

Alkan S, Huang Q, Ergin M, Denning MF, Nand S, Maududi T, Paner GP, Ozpuyan F, Izban KF (2005). Survival role of protein kinase C ( PKC) in chronic lymphocytic leukemia and determination of isoform expression pattern and genes altered by PKC inhibition, Am J Hematol.,79(2): 972-106.

Boehrer S, Nowak D, Hochmuth S, Kim SZ, Trepohl B, Afkir A, Hoelzer D, Mitrou PS, Weidmann E, Chow KU (2005). Daxx over expression in T-lymphoblastic Jurkat cells enhances caspase-dependent death receptor- and drug-induced apoptosis in distinct ways, Cell Signal ., 17 (5): 581-595.

Boellmann F, Guettouche T, Guo Y, Fenna M, Mnayer L, Voellmy R (2004). DAXX interacts with heat shock factor 1 during stress activation and enhances its transcriptional activity. PNAS., 101(12): 4100-4105.

Cermák L, Símová S, Pintzas A, Horejsí V, Andera L (2002). Molecular mechanisms involved in CD43-mediated apoptosis of TF-1 cells. Roles of transcription Daxx expression, and adhesion molecules, J. Biol. Chem ., 277(10): 7955-7961.

Chang CC, Lin DY, Fang HI, Chen RH, Shih HM (2005). Daxx mediates the small ubiquitin-like modifier-dependent transcriptional repression of Smad4, J. Biol. Chem., 280(11): 10164-10173.

Chaudhary P, Sharma R, Sharma A, Vatsyayan R, Yadav S, Singhal SS, Rauniyar N, Prokai L, Awasthi S, Awasthi YC. (2010). Mechanisms of 4-hydroxy-2-nonenal induced pro- and anti-apoptotic signaling. Biochem., 49(29): 6263-6275.
Chen LY, Chen JD (2003). Daxx silencing sensitizes cells to multiple apoptotic pathways, Mol Cell Biol., 23 (20): 7108-7121.

He QZ, Tuo QH, Zeng HC, Zhu BY, Rang WQ, Tang XQ, Liao DF (2010). Daxx mediates oxidized low density lipoprotein induced cholesterol accumulation and apoptosis in macrophages by up regulating Caveolin-1 expression. Progress in Biochem. Biophys., 37(8): 881-890.

Jung YS, Kim HY, Kim J, Lee MG, Pouysségur J, Kim E (2008). Physical interactions and functional coupling between daxx and sodium hydrogen exchanger 1 in ischemic cell death, J. Biol Chem., 283 (2): 1018-1025.

Kenji S, Ryuta M, Tadashi M (2007). Regulation of the STAT3-Mediated Signaling by Daxx, Clin. Immunol., 123: S159.

Khelifi AF, D'Alcontres MS, Salomoni $P$ (2005). Daxx is required for stress-induced cell death and JNK activation, Cell Death Differ., 12 (7): 724-733.

Kitamura T, Fukuyo Y, Inoue M, Horikoshi NT, Shindoh M, Rogers BE, Usheva A, Horikoshi N (2009). Mutant p53 disrupts the stress MAP kinase activation circuit induced by ASK1-dependent stabilization of Daxx, Cancer. Res ., 69(19): 7681-7688.

La M, Kim K, Park J, Won J, Lee JH, Fu YM, Meadows GG, Joe CO (2004). Daxx-mediated transcriptional repression of MMP1 gene is reversed by SPOP, Biochem. Biophys. Res. Comm., 320: 760-765.

Lehembre F, Müller S, Pandolfi PP, Dejean A (2001). Regulation of Pax3 transcriptional activity by SUMO-1-modified PML. Oncogene ., 20: 1-9.

Lin DY, Fang HI, Ma AH, Huang YS, Pu YS, Jenster G, Kung HJ, Shih HM (2004). Negative modulation of androgen receptor transcriptional activity by Daxx, Mol. Cell Biol., 24(24): 10529-10541.

Liu F, Du ZY, He JL, Liu XQ, Yu QB, Wang YX (2012). FTH1 binds to Daxx and inhibits Daxx-mediated cell apoptosis, Mol Biol Rep., 39(2): 873-879.

Meinecke I, Cinski A, Baier A, Peters MA, Dankbar B, Wille A, Drynda A, Mendoza H, Gay RE, Hay RT, Ink B, Gay S, Pap T (2007). Modification of nuclear PML protein by SUMO-1 regulates Fasinduced apoptosis in rheumatoid arthritis synovial fibroblasts, Proc Natl. Acad. Sci. USA., 104(12): 5073-5078

Michaelson JS, Leder $P$ (2003). RNAi reveals anti-apoptotic and transcriptionally repressive activities of DAXX, J Cell Sci ., 116 (2): 34523521.

Muromoto R, Kuroda M, Togi S, Sekine Y, Nanbo A, Shimoda K, Oritani $\mathrm{K}$, Matsuda T (2010). Functional involvement of Daxx in gp130mediated cell growth and survival in BaF3 cells, Eur. J. Immunol., 40(12): 3570-3580.

Park J, Lee JH, La M, Jang MJ, Chae GW, Kim SB, Tak H, Jung Y, Byun B, Ahn JK, Joe CO (2007). Inhibition of NF-Kb acetylation and its transcriptional activity by Daxx, J. Mol. Bio., 368: 388-397.

Schepers H, Geugien M, van der Toorn M, Bryantsev AL, Kampinga HH, Eggen BJ, Vellenga E (2005). HSP27 protects AML cells against VP. 16-induced apoptosis through modulation of p38 and c-Jun, Exp. Hematol., 33(6): 660-670.

Song JJ, Lee YJ ( 2004). Daxx deletion mutant (amino acids 501-625)induced apoptosis occurs through the JNK/p38-Bax-dependent mitochondrial pathway, J. Cell. Biochem., 92(6): 1257- 1270.

Tuo QH, Xiong GZ, Zhu BY,Cao J G, Liao DF (2008). The Effect of Over expressed Daxx in Liver Tumor Cells on The Apoptosis Induced by Oxidative Stress. Progress in Biochem. Biophys ., 35(11): 1270 1275.

Yang X, Khosravi-Far R, Chang HY, Baltimore D (1997). Daxx, a novel Fas-binding protein that activates JNK and apoptosis. Cell, 89 (7): 10672-10676.

Zhang J, Hu YZ, Xueli L, Li S, Wang M, Kong X, Li T, Shen P, Ma Y (2010). The inhibition of CMV promoter by heat shock factor $4 \mathrm{~b}$ is regulated by Daxx, The Int. J. Biochem. Cell Biol., 42: 1698-1707.

Zhang X, Gu L, Li J, Shah N, He J, Yang L, Hu Q, Zhou M (2010). Degradation of MDM2 by the interaction between berberine and DAXX leads to potent apoptosis in MDM2-overexpressing cancer cells,. Cancer. Res., 70(23): 9895-9904.

Zobalova R, Swettenham E, Chladova J, Dong LF, Neuzil J (2008). Daxx inhibits stress-induced apoptosis in cardiac myocytes, Redox Rep., 13(6): 263-270. 